\title{
Foreign Fiber Detection in Cotton using HSI Approach for Industrial Automation
}

\author{
Eyasmina Parveen \\ M.Tech in NIT Arunachal Pradesh \\ Dept. Mobile Communication and \\ Computing (MCC)
}

\author{
Koj Sambyo \\ Asst. Professor and HOD of \\ Computer Science and \\ Engineering \\ NIT, Arunachal Pradesh
}

\author{
Kausar Ahmed \\ M.Tech in NIT, Arunachal \\ Pradesh \\ Dept. Mechanical Engineering
}

\begin{abstract}
Foreign fibres in cotton have seriously affected the quality of cotton goods. The classification and identification of foreign fibres in cotton is the basis of automated inspection of foreign cotton fiber. Application of Support Vector Machine (SVM) for analysis of foreign fibres in cotton. One of the advantages of SVM is that, with limited training data, it can generate results similar or better than other methods. The SVM algorithm is used for automated object detection and characterization. Specifically, the SVM is applied in its basic nature as a binary classifier where it classifies two different folders one having the cotton image and second one contains thesupervised images. The algorithm aims at effectively detecting an object from its background with the minimum training data. The synthetic image containing noises is used for algorithm testing. Furthermore, it is implemented to perform remote sensing image analysis such as identification of Island vegetation, water body, and oil spill from the satellite imagery. It is indicated that SVM provides the fast and accurate analysis with the acceptable result. Furthermore the introduction of $\mathrm{CNN}$ has been apply to the proposed technique to this presentation offers a new method for using templates that match the templates of Convolution Neural Network. The CNNs are running simultaneously to train template images. All Information about image template is send to neural network that has been convoluted the image with saved template in very fast manner. It is accuracy while scanning an image and convolutes the pixel with pure image pixel and finds the exact difference in between them. The test picture is a cotton production image containing the foreign image and find out the impurities in running a production line of cotton with high accuracy. This thesis has a complete demonstration of detection system for the foreign fibers in raw cotton. The accuracy of the result is evaluated by the regression analysis of the outcomes like the time of simulation and the size of pictures in pixel speared over the horizontal and vertical area. The results of the proposed analysis have shown that the time is unpredictable for the given set of images. The logistic regression has exponential nature that suggests that the simulation time is drastically increases as the area of cotton size increases. The accuracy provide by proposed technique is very near to hundred percent.
\end{abstract}

\section{General Terms}

Recognition, Color Space , Image Processing, Algorithm, Simulation.

\section{Keywords}

Foreign Fiber, Image Pixels, Template Matching Process, Convolution Neural network.

\section{INTRODUCTION}

Features of respective image template can be used to identify proper analysis. The study of features of a cotton fibers is one of the important aspects of our research. The most popular clothes in the world is cotton. It is used to produce natural fiber and various types of cotton fabric. The quality of cotton fiber deteriorates due to various foreign fibres, such as plastic film, nylon, jute, dry cotton, bird feathers, paper and silk, nylon, polypropylene and others. Contamination of raw cotton can be performed on all steps, from the initial phase to last phase. Since cotton impurities was selected with hand by rural women like human hair, pollution caused by pieces. In addition of the foreign fiber including storages, plastic film, jute, hair, polypropylene gum and rubber clock is a serious threat to the textile and cotton industries. These selections of impurities and type of impurities are first determinate with their size, shape and colour[2].

\subsection{Challenges}

There were several challenges encountered when attempting to develop our algorithm, because cotton images can demonstrate a wide degree of variation in both shape and texture. Appearance variations are caused by individual differences, the detection of foreign fibers due to changes in shape and size, as well as lighting variations. These issues are explained more in the following points:

- The foreign fiber detection problem is challenging problems as depends on many factors, some of them are visual and many others are non-visual such as size, shape, colours and numbers.

- The visual features that can help in evaluating shape, size, and colour of foreign fibers are affected by position \& orientation.

- The difficulty of acquiring large-scale databases, which covers enough template range with various images of both pure cotton and foreign fibers, makes the estimation tasks more difficult to achieve[1].

\subsection{Process Overview}

Modern cotton processing is very industrialized production line automation[1].Cotton is separated from raw seeds and then process to processing units. One certain cleaning and elimination of pollution can be occurring during flushing. Cotton is usually in raw cotton seeds[2]. That's it we mainly target the yarn production level Application Sync Detection Computer Protection system. In the yarn production line, cotton can be refined with the process of filter line and moved by blowing through the sledge. Automatic pollution detection and removal the system is integrated on this line. Insert the camera constantly monitor the location of mobile cotton and collect photos. 


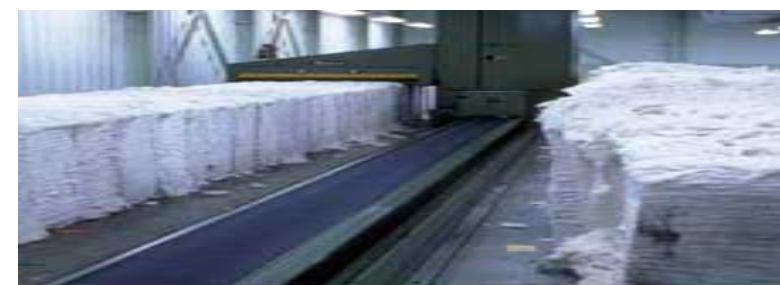

Figure 1: Selected Cotton is delivered in a cup for yarn production.

\subsubsection{Image processing system suitable for} analysis of foreign fibers.

When the picture apply through algorithm is completed, a portion of cotton is often detected due to the air turbine flow is much faster than the capturing capacity of camera during the processing of foreign fibers. Due to the fast scanning process it is some time not captures the foreign elements. Due to implementation of larger data set the problem can be short out.

\subsection{Similar work on foreign fibers detection}

We can classify computer processing methods for sorting the impurities of cotton. Based on colour/intensity detection method, the pixel value of image has been modulated so that the external elements rather than the cotton have been detected easily. Most methods are available, some pictures are pre-processed after the main deal in the steps, the usual threshold determines which pixels belong to it contaminants. Post-processing steps such as morphological filters, area increase, and connection part analysis and so on are also com monly used reference [6]. One is given a good overview of the system starting with the sensor inspiration, blowing away foreign objects. Short the review also found [7]. Research has taken place the efficiency of different wavelengths [8][9]. Before cotton fabric can be very diverse, a wide range ,the strategy may need to cover all categories of the actual system of contaminants.

\subsection{Selection of Colour Space}

\subsubsection{RGB Color Space}

The RGB color model is the most basic and the most used hardware for image processing. The cotton image form using RGB has also very suitable for detection process of hardware. The RGB model uses the three basic components of Red, Blue and Green for representation of the color. In this system, all colors are justified in the RGB color bar. The RGB color range, however has a major disadvantage; the most important is that it is not intuitive, so it is difficult to know the value of a cognitive property of its RGB value. The RGB has very distinguish feature that is incorporated with detection process of any machine learning algorithms.

\subsubsection{HSI Color Space}

HSI means hue, saturation and intensity. The HSI model differentiates extra components from image screens into the image. Therefore, it is a tool for assisting imaging with a variety for colour modeling. Currently, in many computer visual systems, many computer divisions apply. An example of computer vision systems, the real-time automatic detection and identification system are HSI (for example Hue,saturation and intensity) and others like RGB. This model applies to the detection of foreign fibers[9].

\subsubsection{YCbCr Color Space}

External cotton fiber analysis algorithm in the following model compares HSI and YCbCr color. The advantage of the $\mathrm{YCbCr}$ model is that it can work with chromium lighting and exposure, in particular by using useful information from the original image as much as possible. The original image is in the form of RGB, so the color scheme must change. There are many colors, where luminance and crumbling components are separated, such as YCbCr, HSV etc. This methodology accepts the $\mathrm{YCbCr}$ color scheme. Under this scheme the pixel value of RGB space into luminance Y,chrominance of blue $\mathrm{Cb}$, and chrominance of red $\mathrm{Cr}$ in the $\mathrm{YCbCr}[9]$.

The conversion formula used is:

$\mathrm{Y}=16+(65.481 \mathrm{R}+128.553 \mathrm{G}+24.966 \mathrm{~B})$

$\mathrm{Cb}=128+(-37.797 \mathrm{R}-74.203 \mathrm{G}+112.0 \mathrm{~B})$

$\mathrm{Cr}=128+(112.0 \mathrm{R}-93.786 \mathrm{G}-18.214 \mathrm{~B})$

\section{TEMPLATE MATCHING PROCESS}

A template matching process uses pixels, samples, models or textures as pattern. The recognition function computes the differences between these features and the stored templates. It uses correlation or distance measures. Although the matching of $2 \mathrm{D}$ images was the early trend, now-a-days $3 \mathrm{D}$ templates are more common. The 2D approaches are very sensitive to orientation or illumination changes. One way of addressing this problem is using Elastic Bunch Graphs to represent images. Each subject has a bunch graph for each of its possible poses. Image features are extracted from the test image to form an image graph. This image graph can be compared to the model graphs, matching the right class. The introduction of 3D models is motivated by the potential ability of three dimensional patterns to be unaffected by those two factors. The problem is that $3 \mathrm{D}$ data should be acquired doing 3D scans, under controlled conditions. Moreover, in most cases requires the collaboration of the subject to be recognized.Therefore, in applications such as surveillance systems, this kind of 3D data may not be available during the recognition process. This is why there is tendency to build training sets using 3D models, but gathering 2D images for recognition. Techniques that construct 3D models from 2D data are being developed in this context[11].

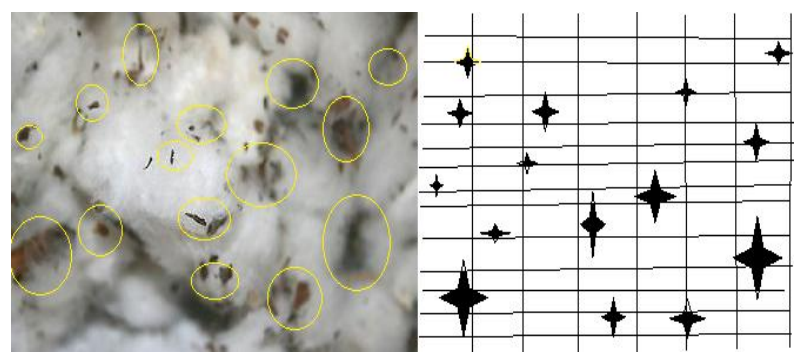

Fig 2.1: Explain how the pixel in grid based image pattern

\section{TEMPLATE MATCHING ALGORITHM UNDER CONVOLUTION NEURAL NETWORK}

One of the basic ways to match a template is to use a wrapper mask (template) designed specifically for the specific functionality of the search image we want to detect. The technology can be easily executed on gray images or edge images. Where the image structure matches the mask 
structure, the package output is higher, with larger image values multiplied by larger mask values.

A pixel in the search image with coordinates $\left(\mathbf{x}_{\mathbf{s}}, \mathbf{y}_{\mathbf{s}}\right)$ has intensity $\mathbf{I}_{\mathbf{s}}\left(\mathbf{x}_{\mathbf{s}}, \mathbf{y}_{\mathbf{s}}\right)$ and a pixel in the template with coordinates $\left(\mathbf{x}_{\mathbf{t}}, \mathbf{y}_{\mathbf{t}}\right)$ has intensity $\mathbf{I}_{\mathbf{t}}\left(\mathbf{x}_{\mathbf{t}}, \mathbf{y}_{\mathbf{t}}\right)$. Thus the absolute difference in the pixel intensities is defined as Diff $\left(\mathbf{x}_{\mathbf{s}}, \mathbf{y}_{\mathbf{s}}, \mathbf{x}_{t}\right.$, $\left.\mathbf{y}_{\mathrm{t}}\right)=\left|\mathbf{I}_{\mathrm{s}}\left(\mathbf{x}_{\mathrm{s}}, \mathbf{y}_{\mathrm{s}}\right)-\mathbf{I}_{\mathrm{t}}\left(\mathbf{x}_{\mathrm{t}}, \mathbf{y}_{\mathrm{t}}\right)\right|$.

$\operatorname{SAD}(\mathrm{x}, \mathrm{y}) \sum_{i=0}^{\text {trows }} \sum \operatorname{diff}(x+i, y+j, i, j)$

The mathematical representation of the idea about looping through the pixels in the search image as we translate the origin of the template at every pixel and take the SAD measure is the following:

$$
\sum_{i=0}^{\text {sraw }} \sum_{y=0}^{\text {scols }} S A D(x, y)
$$

$S_{\text {rows }}$ and $S_{\text {cols }}$ represent the rows and columns of the search image, $\mathrm{T}_{\text {rows }}$ and $\mathrm{T}_{\text {cols }}$ represent the row and column in the template respectively. In this method, the lowest SAD estimates the best location of the template within the search image. This method is easy to implement and understand, but it is one of the slowest methods. To achieve fast and reliable detection of cotton few fast learning algorithm is apply over the pixel detection and matching process of pattern [10].

\section{SIMULATION WORK}

This paper test on few pictures of cotton contains foreign elements of different shape and size as shown below

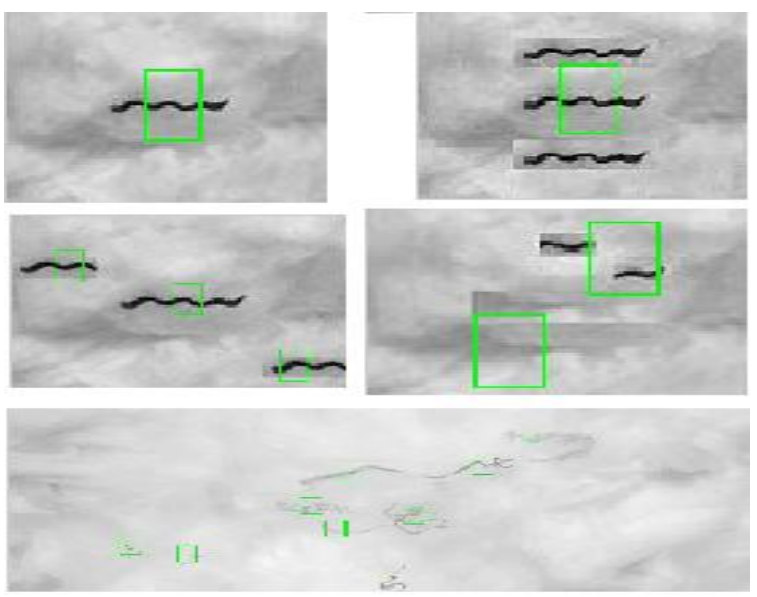

Fig 4.1: Showing the detection of foreign fibers in various pictures.

Table 4.1: Simulation Time with image size

\begin{tabular}{|l|l|l|}
\hline $\begin{array}{l}\text { JPG type } \\
\text { Image }\end{array}$ & $\begin{array}{l}\text { Size of } \\
\text { Image }\end{array}$ & Run Time \\
\hline IM1 & $394 * 297$ & 25.171159 seconds \\
\hline IM2 & $400 * 299$ & 39.059348 seconds \\
\hline IM3 & $400 * 300$ & 19.720187 seconds \\
\hline IM4 & $400 * 299$ & 41.145979 seconds \\
\hline IM5 & $107 * 71$ & 729.939435 second s \\
\hline IM6 & $151 * 103$ & 739.733252 seconds \\
\hline IM7 & $100 * 74$ & 78.483827 seconds \\
\hline
\end{tabular}

\begin{tabular}{|c|c|c|}
\hline IM8 & $100 * 75$ & 84.375377 seconds \\
\hline IM9 & $100 * 75$ & 776.946775 seconds \\
\hline IM10 & $92 * 72$ & 946.153321 seconds \\
\hline IM11 & $100 * 75$ & 93.474568 seconds \\
\hline IM12 & $200 * 149$ & 726.856718 seconds \\
\hline IM13 & $612 * 316$ & 2613.554263 seconds \\
\hline IM14 & $82 * 125$ & 92.293305 seconds \\
\hline IM15 & $117 * 75$ & 788.713112 seconds \\
\hline IM16 & $117 * 75$ & 715.927785 seconds \\
\hline IM17 & $150 * 104$ & 262.320985 seconds \\
\hline IM18 & $150 * 113$ & 126.564441 seconds \\
\hline IM19 & $150 * 77$ & 264.749882 seconds \\
\hline IM20 & $250 \times 129$ & 831.777286 seconds \\
\hline IM21 & $287 * 190$ & 7.702109 seconds. \\
\hline IM22 & $289 * 294$ & 16.666348 seconds. \\
\hline IM23 & $219 * 278$ & 8.360727 seconds. \\
\hline IM24 & $101 * 75$ & 522.083893 seconds. \\
\hline IM25 & $99 * 74$ & 110.340971 seconds. \\
\hline IM26 & $98 * 73$ & 555.019722 seconds. \\
\hline IM27 & $103 * 77$ & 627.730198 seconds. \\
\hline IM28 & $195 * 145$ & $\begin{array}{l}1478.361938 \\
\text { seconds. }\end{array}$ \\
\hline IM29 & $151 * 113$ & 1063.502750 seconds \\
\hline IM30 & $257 * 193$ & 67.181400 seconds. \\
\hline IM31 & $150 * 113$ & 222.446148 seconds. \\
\hline IM32 & $299 * 224$ & 211.756350 seconds. \\
\hline IM33 & $53 * 39$ & 55.911605 seconds. \\
\hline IM34 & $302 * 225$ & 959.809065 seconds. \\
\hline IM35 & $203 * 151$ & 278.065141 seconds. \\
\hline
\end{tabular}

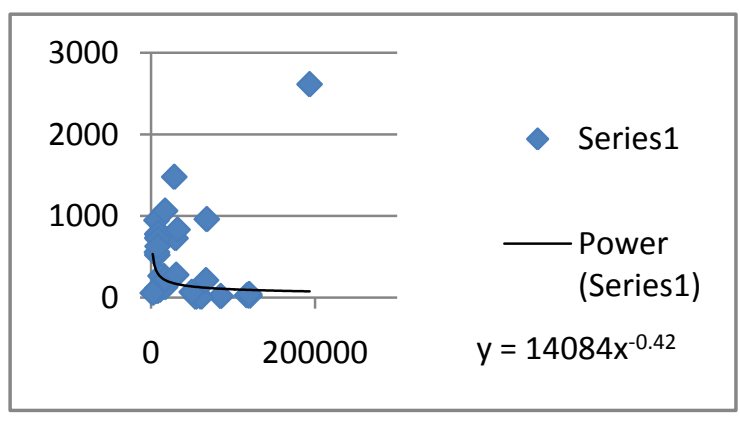

Fig 4.2: Line of regression

As per various picture were experimented through simulation model and it is found that the size of picture don't depend upon the execution time. It depends upon the location of foreign fibers and the number of their occurrence. The detection of successive pictures found to be great and detect 
the foreign elements every time. This graph is experimental presentation of execution of time and area which is the basic information of data trends. This shows expectancy of time of new picture through the line.

\section{CONCLUSION}

Template matching has been a constant challenge in the field of vision on a computer for many decades and is still unresolved. With the introduction of depression networks, a new method of coordinating project templates has been developed. The method chosen for this article is to use adaptation methods to learn about local information template. This new image detection method has been adopted more effectively with foreign fibers detection on the layer of cotton. Image as a templates, image matching up for test image both taken for the simulation in MATLAB. One behaves as a data base and one as a test element that is match up with the data base of the templates. The main step of this method is to recognize foreign fibers on cotton fibers. The size of all foreign fiber images is monochromatic. An object element occupies only a very small portion of the entire volume image. It is very hard to find such small elements of fiber on the layout of cotton.

A various image of cotton and non cotton images has been saved in separate folder then the test picture has been taken to match up with the data base. Then SVM will find the exactness of matching with templates images saved in folders. The color image segmentation introduced with the regional growth algorithm has better adaptability. The effectiveness of foreign fiber detection algorithms has been proven on various test images. Some quantitative image segmentation methods are used to evaluate the results. Future prospective of this technique is to implement it as a commercial level to enhance the automatization problems in mass production of cotton. This will also enhance the quality of cotton production level.

At last this paper introduces best way to find the unwanted element through SVM and Convolution Neural network. This has experimented on almost 35 images with time and area. This find

\section{FUTURE SCOPE}

We can explore more algorithms and techniques for the feature extraction and classification of cotton contaminants to further improve the accuracy of the identification system.

We can further improve the system by reducing the complexity. The main objective could be to find the best algorithms which optimize the performance and complexity.

The accuracy of classifier can also be enhanced by using more and equal number of training pattern.

\section{ACKNOWLEDGEMENTS}

I take this opportunity to express my heartfelt gratitude and sincere thanks to my project supervisor Dr. Koj Sambyo Assistant Professor and Head of the Department of Computer science Engineering, National Institute of Technology, Arunachal Pradesh for his constant guidance, suggestion and gracious encouragement at my very crucial stage of this dissertation work without him it could not become successful.

I sincerely thank all those people who gave me suggestions while working on this project. I am highly thankful to Amit Kumar, my parents, friends, and faculty members who were there all the time and gave support to my endeavor from behind the scene.

\section{REFERENCES}

[1] Kadir A. Peker and Gökhan Özsar1,"Contaminant and Foreign Fiber Detection in Cotton Using Gaussian MixturModel,"@ DOI: 10.1109/ICAICT.2014.7035922, October 2014, IEEE 8th International Conference .

[2] Ling Ouyang, Hongtao Peng, Dongyun Wang,Yongping Dan and Fanghua Liu,"Supervised Identification Algorithm on Detection of Foreign Fibers in Raw Cotton," IEEE 2012 24th Chinese Control and Decision Conference (CCDC), pp-2636-2639, 2012.

[3] Dongyun Wang,Hongtao Peng,Yongping Dan, Fanghua Liu and Liusong Wang. "Algorithm on Detection and Identification of Foreign Fibers in Raw Cotton," IEEE Proceedings of the 2011 International Conference on Advanced Mechatronic Systems, Zhengzhou, China, August 11-13, pp-43- 46, 2011.

[4] Chengliang Zhang, Xianying Feng, Lei Li and Yaqing Song, "Detection of Foreign Fibers in Cotton on the Basis of Wavelet," IEEE 2010 2nd International Conference on Signal Processing Systems (ICSPS), pp304-308, 2010.

[5] Jia DongYao , Ding Tian Huai. "Detection of Foreign Fibers in Cotton Using NIR Optimal Wavelength Imaging," IEEE Imaging and Imaging Applications, pp751-752, 2004.

[6] Chen Yajun, Zhang Erhu, Kang Xiaobing. "Divisional Velocity Measurement For High-Speed Cotton Flow Based on Double CCD Camera and Image CrossCorrelation Algorithm," The 11th IEEE International Conference on Electronic Measurement \& Instruments, pp-2020-206,2013.

[7] Zhang Qing et. al. "Design of Raw Cotton Foreign Fibers Detecting and Clearing On Line System," The 7th International Conference on Computer Science \& Education (ICCSE 2012) July 14-17, Melbourne, Australia, pp-1223-1225, 2012.

[8] Tingting Xie et. al. A Method for "Detection of Foreign Body in Cotton Based on RGB Space Model," IEEE, pp31-33, 2011.

[9] Aditi Sachar and Sugandha Arora,"Cotton Contaminants Detection and Classification using $\mathrm{HSI}$ and $\mathrm{YCbCr}$ model,'International ISSN:2278-067X,Volume 1,Issue 10 (June 2012),PP.2935.

[10] Dr.S.Vijayarani and Ms.A.Sakila,Template "Matching Technique for Searching Words in Document Images,'International Journal on Cybernetics \&Informatics(IJCI)Vol,4,No,6,December 2015.

[11] Zhong Jin ,Zhen Lou,Jingyu Yang, Quansen Sun, "Face detection using template matching and skin color information," Neurocomputing 70 (2007) 794-800. 